\title{
Extreme Ultraviolet Radiation Measurement for Planetary Atmospheres/Magnetospheres from the Earth-Orbiting Spacecraft (Extreme Ultraviolet Spectroscope for Exospheric Dynamics: EXCEED)
}

\author{
Ichiro Yoshikawa • Kazuo Yoshioka • Go Murakami • Atsushi Yamazaki • \\ Fuminori Tsuchiya • Masato Kagitani · Takeshi Sakanoi • Naoki Terada • \\ Tomoki Kimura • Masaki Kuwabara • Kuto Fujiwara • Tomoya Hamaguchi • \\ Hiroyasu Tadokoro
}

Received: 7 June 2014 / Accepted: 23 July 2014 / Published online: 8 October 2014

(C) The Author(s) 2014. This article is published with open access at Springerlink.com

\begin{abstract}
The Sprint-A satellite with the EUV spectrometer (Extreme Ultraviolet Spectroscope for Exospheric Dynamics: EXCEED) was launched in September 2013 by the Epsilon rocket. Now it is orbiting around the Earth $(954.05 \mathrm{~km} \times 1156.87 \mathrm{~km}$ orbit; the period is 104 minutes) and one has started a broad and varied observation program. With an effective area of more than $1 \mathrm{~cm}^{2}$ and well-calibrated sensitivity in space, the EUV spectrometer will produce spectral images (520-1480 $\mathrm{A}$ ) of the atmospheres/magnetospheres of several planets (Mercury, Venus, Mars, Jupiter, and Saturn) from the Earth's orbit. At the first day of the observation, EUV emissions from the Io plasma torus (mainly sulfur ions) and aurora $\left(\mathrm{H}_{2}\right.$ Lyman and Werner bands) of Jupiter have been identified. Continuous 3-month measurement for Io's plasma torus and aurora is planned to witness the sporadic and sudden brightening events occurring on one or both regions. For Venus, the Fourth Positive $\left(\mathrm{A}^{1} \Pi-\mathrm{X}^{1} \Sigma^{+}\right)$system of $\mathrm{CO}$ and some yet known emissions of the atmosphere were identified even though the exposure was short (8-min). Long-term exposure from April to June (for approximately 2 months) will visualize the Venusian ionosphere and tail in the EUV spectral range. Saturn and Mars are the next targets.
\end{abstract}

Keywords EUV radiation · Plasma emissions · Jupiter · Mars · Venus · EXCEED/SPRINT-A

I. Yoshikawa $(\bigotimes) \cdot$ M. Kuwabara $\cdot$ K. Fujiwara $\cdot$ T. Hamaguchi

Department of Earth and Planetary Science, The University of Tokyo, Tokyo 113-0033, Japan e-mail: yoshikawa@eps.s.u-tokyo.ac.jp

K. Yoshioka · G. Murakami · A. Yamazaki · T. Kimura

Japan Aerospace Exploration Agency, Institute of Space and Astronautical Science, Sagamihara, Kanagawa 252-5210, Japan

F. Tsuchiya $\cdot$ M. Kagitani $\cdot$ T. Sakanoi $\cdot$ N. Terada

Planetary Plasma and Atmospheric Research Center, Tohoku University, Sendai 980-8578, Japan

H. Tadokoro

School of Computer Science, Tokyo University of Technology, Hachioji, Tokyo 192-0982, Japan 


\section{Introduction: Mission Background}

Japanese intensive exploration of space began with the launch of the first Earth-orbiting spacecraft, OHSUMI. It had a mass of only $24 \mathrm{~kg}$, and today this spacecraft might be classified as a micro-satellite. After the success, the Institute of Space and Astronautical Science (ISAS) has built and launched more than 25 satellites for science and/or engineering purposes. Traditionally, many space researchers have carried out sounding-rocket experiments to have an access-to-space and to provide "hands-on" training ground for long-term missions. Sounding-rocket experiments were planned quickly and executed at low costs, and they provided frequent opportunities for space researches. However, in the 1990s, a solid propulsion rocket (M-V) developed by ISAS had been used as one of the powerful vehicles but at higher cost. As a result, the number of space programs decreased.

On the other hand, a series of small scientific satellites, which ISAS has started to develop, based on the concept 'cheaper and faster realization of unique space experiments' as a complementary program of mainstream scientific satellites. ISAS has released the plan to launch some small satellites over 5 years (Fukuda et al. 2008). The mass of the satellites is approximately $350 \mathrm{~kg}$. This approach allows one to significantly reduce the time required to obtain science output. Moreover, the shorter development period will reduce overall cost. ISAS has built the standard bus architecture, where the bus and the payload are clearly separated in a modular manner. The series of the spacecraft employing standard bus architecture is named the Small scientific satellite Platform for Rapid Investigation and Test (Sprint series).

Fifteen working groups to propose small scientific satellites in this category were constituted under the Steering Committees of Space Science and Space Engineering in ISAS. Our EXCEED mission (EXtreme ultraviolet speCtroscope for ExosphEric Dynamics) was one of the proposals and was selected as the first program in 2007. It was based on our technological advances in the extreme ultraviolet (EUV) spectral range during the past two decades (e.g. Yoshikawa et al. 1997, 2001, 2005, 2010b; Murakami et al. 2013; Yoshioka et al. 2012; Chassefière et al. 2010). We have enabled quantitative spectroscopy to provide detailed information on neutral and ionized gases of atmospheric and astrophysical interest. There are several fundamental reasons why technological advances which have opened the EUV spectral range to investigate are important: (1) strong resonance transitions of the most abundant atmospheric and exospheric elements, e.g., hydrogen, helium, and oxygen have emission lines in the spectral range shortward of $1500 \AA$ (Nakamura et al. 1999; Yoshikawa et al. 2008; Yamazaki et al. 2002), (2) for solar planets, interactions among solar wind, the magnetospheres, the exospheres, ionospheres, and atmospheres give rise to diagnostic EUV emission on a global scale (Yoshioka et al. 2011, 2013; Yamazaki et al. 2003), (3) many of the primary astrophysical interaction processes occur in the energy equivalent temperature from $10^{4}$ to $10^{6}$ Kelvin, producing transitions in the EUV (Yamazaki et al. 1996).

Our ultimate goal in this mission is to characterize the planetary atmospheres/magnetospheres. Planetary magnetospheres are characterized by the strength of the intrinsic dipole magnetic moment, planetary rotation period, and plasma sources. Compared with the Earth, Venus and Mars have a weak magnetic moment in the core and their atmospheres are forced to directly interact with the solar wind. The interaction results in loss of plasmas in the upper atmospheres to interplanetary space. The loss of atmospheric gases to space is thought to make a significant contribution to the evolution of the planetary atmosphere. On the other hand, Jupiter and Saturn have a strong magnetic moment, internal plasma sources (Io and Enceladus tori, respectively), and fast planetary rotations. Consequently, plasma convection in these magnetospheres is dominated by azimuthal flow around the planet, which is in 
Fig. 1 Bold lines indicate nominal durations for observation of targets executed by EXCEED. The Sun-spacecraft-planet angle (elongation angle of planets) as a function of date is plotted. Initial operation and joint observation periods are also indicated. The lower limit of the elongation is $20^{\circ}$ for the observation to avoid direct solar illumination to the inside of the telescope. This limit shortens the duration of the Mercury observation

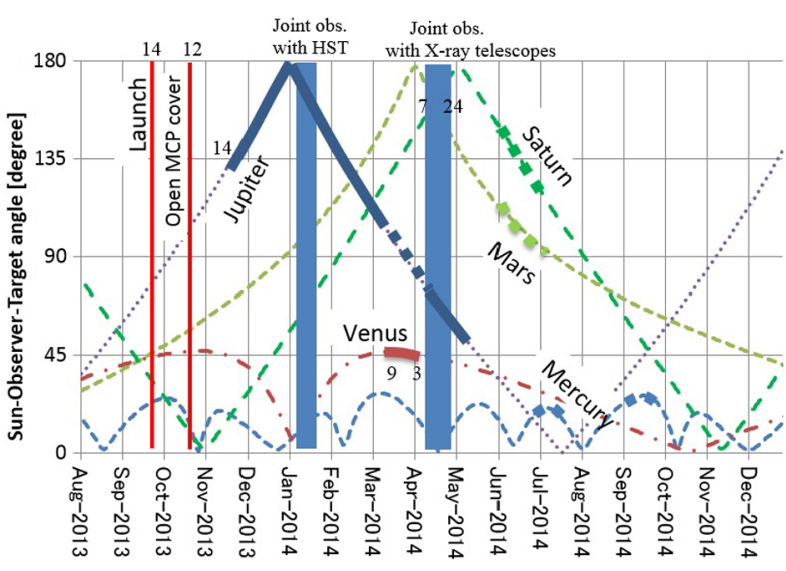

contrast to the terrestrial magnetosphere, where the plasma flow is mainly driven by the solar wind.

Our science instrument (EXCEED) is solely boarded on the SPRINT-A spacecraft built by ISAS/JAXA. EXCEED on SPRINT-A is the world's first observatory in space observing planets, Venus, Mars, Jupiter, and Saturn. Spectroscopic imaging in the EUV spectral range, which cannot be performed on the ground, allows us to collect information on the planetary atmospheres and magnetospheres. Here we present the instrument specifications with inflight calibration and the initial results of the observations.

\section{Observation Schedule}

Figure 1 shows the observation schedule. The $X$-axis presents the month and year, the $Y$-axis is the Sun-Earth-planet angle (elongation peeling angle).

After the launch of the spacecraft (the details are shown later), we had to wait until the outgassing from the spacecraft dried up. On October 12th, we opened the door of the detector chamber by activating the paraffin actuator. We applied the high voltage onto the detector unit and started to increase the level of high voltage gradually until 17th November. It reached the high-voltage level by $3.55 \mathrm{kV}$ on November $17 \mathrm{th}$.

The first lights of Jupiter and Venus were obtained in November 18th. We started the continuous observation of Jupiter until the beginning of March. During the first and second weeks of 2014, joint observation with Hobble Space Telescope (HST) was successfully made. EXCEED changed the target to Venus in April and has taken a long time for the exposure, except the campaign period for the observation of Jupiter with $X$-ray telescopes (Suzaku, Chandra, and XMM). In this joint observation, we will observe the Jupiter intermittently for a couple of weeks.

From June 2014, we will face the challenge to observe Saturn looking small, Mars looking less bright, and with Mercury close to the Sun. The observation target was primarily chosen due to the elongation angle.

\subsection{First Target: Jupiter}

The interaction of planetary magnetosphere with solar wind generates a polar arc (aurora) on Earth, Jupiter, and Saturn. The Jovian magnetosphere with the strongest magnetic field in the solar system $(10,000$ times stronger than that of the Earth) and with planetary rotation at 
Fig. 2 The black dots show the total emitted power from the Io torus and the Jovian aurora (red dots) over the 640-1180 $\AA$ wavelength range during the Cassini spacecraft flyby of Jupiter. Sudden brightening events in IPT were coincident with auroral brightenings (vertical green lines). (A.J. Steffl, personal communication)

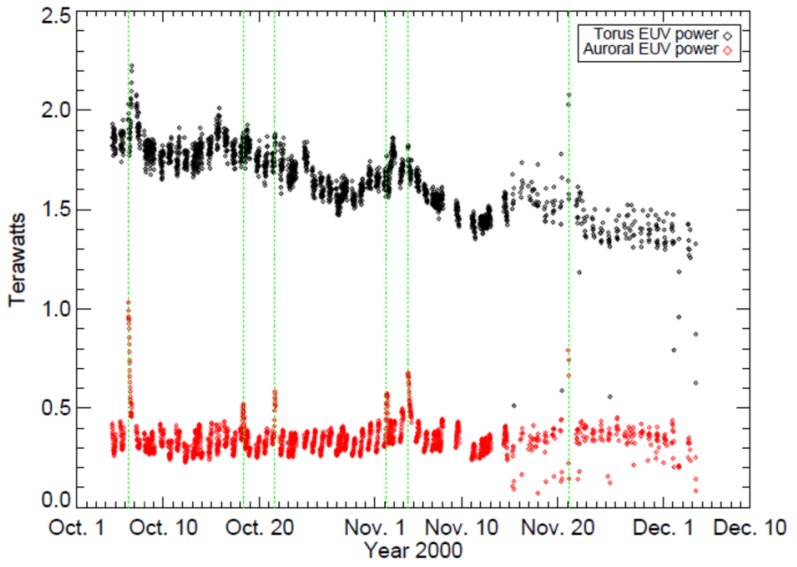

a high speed of ten hours per cycle is known to be dominated by the plasma flow co-rotating around the planet. Co-rotating regions are common in the magnetospheres of magnetized bodies (Mercury, Earth, Jupiter, and Saturn). The terrestrial magnetosphere has a co-rotating region named the plasmasphere and it is strongly affected by the solar wind. On the other hand, the strength of Jovian co-rotation induced electric field is orders of magnitude higher than that generated by the solar wind, therefore plasmas in the inner magnetosphere inside a radial distance of 10-20 $R_{J}\left(R_{J}\right.$ : Jovian radius $\left.=71,492 \mathrm{~km}\right)$ are considered to co-rotate with the planetary rotation. As a result, it has been thought that transports of plasma and energy across the azimuthal direction are difficult in the Jovian magnetosphere, unlike the Earth.

Volcanic gases $\left(\mathrm{SO}_{2}, \mathrm{SO}\right)$ released from Io are dissociated and ionized mainly by electron impacts. More than $1000 \mathrm{~kg}$ of neutral gases from Io's atmosphere are ionized per second and produce a dense (approximately $2000 \mathrm{~cm}^{-3}$ ) torus of electrons, sulfur, and oxygen ions, trapped in Jupiter's strong magnetic field. While in situ measurements of the Io plasma torus by the Voyager and Galileo spacecraft (Frank and Paterson 2000; Bagemal 1985) and remote-sensing observations from the ground and from space-based UV telescope have characterized the density, temperature and composition of the plasma as well as the basic structure (Broadfoot et al. 1981; Steffl et al. 2004a, 2004b; Thomas et al. 2003), the temporal variability of the torus remains poorly determined.

Most interestingly, the UV spectrometer on the Cassini spacecraft witnessed a puzzling phenomenon. Figure 2 shows the brightnesses of Io plasma torus and Jupiter aurora as a function of date. Sometimes both regions were brightened almost simultaneously, but sometimes one was. How does the energy transport from one to the other so fast across the azimuthal flow of the inner magnetosphere? There are over 200 individual radiative transitions from the major ion species present in the torus that lie in our spectral range according to the CHIANTI atomic physics database. The spectrum is sensitive to electron temperature $(>5 \mathrm{eV})$. With the EUV spectrum and the CHIANTI atomic physics database, we can extract the electron temperature and ion mixing ratios based on the spectral diagnosis method (Steffl et al. 2004a, 2004b; Yoshioka et al. 2011, 2013). The distribution and temporal variation of hot electrons is a potential target for measurement to clarify this issue (Yoshioka et al. 2011).

\subsection{Second Target: Venus and Mars}

Due to the thick atmosphere and the intrinsic magnetic moment, our Earth sustains the environment in which a variety of creatures have lived. In contrast, although Venus and Mars 
are Earth-like planets, with temperatures and atmospheric pressures of $-40{ }^{\circ} \mathrm{C}$ and $750 \mathrm{~Pa}$ on Mars and $400{ }^{\circ} \mathrm{C}$ and $9000 \mathrm{kPa}$ on Venus, these two planets have environments that are thoroughly exotic compared with that of the Earth. Since Mars and Venus have weak intrinsic magnetic dipole moments, their atmospheres are considered to have escaped or been escaping to space due to the solar wind interaction.

One of the most important studies in planetary science is the characterization of the formation of terrestrial planets' environments and their divergent evolutions. The existence of $\mathrm{CO}_{2}$ and $\mathrm{N}_{2}$ in the past atmosphere indicates a mild climate in the planet. If we measure how much atmosphere is lost to space constantly, it will allow us to estimate the total loss that may have occurred during billions of years, possibly making Mars red and dry as it is today. EXCEED will witness the solar wind interaction with planetary atmospheres by the charge exchange process in the dayside (solar wind ions charge-exchanges atmosphere of planets). The distribution of escaping ionospheric ions to space, forming an ionospheric tail, is imaged by solar resonantly scattering emissions in the EUV spectral range $\left(\mathrm{O}^{+}, \mathrm{C}^{+}\right.$, and $\mathrm{N}^{+}$, the necessary exposure period is discussed in Sect. 3.3). No instrument has ever been flown to measure the cumulated loss of atmosphere. This measurement is essential to understand why Earth-like planets could not become more exactly like Earth.

Collaboration with other space missions is helpful to understand the total escaping flux and the currently operating mechanism for it. In the past, many satellites investigated the plasma environment of a planet without measurement cumulated amount/loss of atmosphere. The MAVEN spacecraft with a magneto-sensor, plasma analyzer, and UV spectrometer, intended to study escape of Mars's atmosphere, was launched in 2013 and will arrive at Mars in late 2014. It will be just after EXCEED starts the observation of Mars. In situ and local remote-sensing measurements on MAVEN will bring information on particle energy, velocity, density and their variation versus solar wind condition. Simultaneous measurements of cumulated amount/loss of atmosphere by EXCEED and particle characteristics by in situ (local remote-sensing) instrument on orbiter can fill the gap each other.

\subsection{Third Target: Mercury and Saturn}

Mercury, like the Moon, has no stable atmosphere. The gaseous environment is described as an exosphere. The mean free paths of the constituents are large and the total column density is less than $10^{12} \mathrm{~cm}^{-3}$. The existence of seven elements, $\mathrm{O}, \mathrm{H}, \mathrm{He}, \mathrm{Na}, \mathrm{K}, \mathrm{Ca}$, $\mathrm{Si}$, and $\mathrm{Mg}$ has been identified, the first three elements by Mariner-10, the next three by ground-based telescopes, the last two by Messenger observations (Potter and Morgan 1985; Kameda et al. 2007; Bida et al. 2000; Vervack et al. 2010). It is highly probable that other elements, such as $\mathrm{C}, \mathrm{N}, \mathrm{S}, \mathrm{OH}, \mathrm{Al}$, and Fe are also present. The exosphere of Mercury will be further investigated by BepiColombo mission (Chassefière et al. 2010; Yoshikawa et al. 2010a). EXCEED will search for predicted species, $\mathrm{C}$ and $\mathrm{N}$ have emission lines in EUV, not previously detected, as well as new species.

Saturn is the second largest planet in our solar system; it is surrounded by numerous rings and satellites. The Cassini spacecraft made observations in the magnetosphere after the arrival at Saturn on 30 June 2004. Saturn's magnetic dipole moment is $0.21 \mathrm{GRs}^{3}$ (1 Rs = $60,268 \mathrm{~km}$ ), and the magnetic field intensity on the surface is similar to Earth's one. The magnetic field axis is almost aligned with the rotation axis. Saturn's magnetosphere shows both internal (e.g., Hill et al. 2005; Burch et al. 2007) and solar wind controls (e.g., Kurth et al. 2005; Mitchell et al. 2005).

Saturn's inner magnetosphere is dominated by water group neutrals $\left(\mathrm{H}_{2} \mathrm{O}, \mathrm{OH}\right.$, and $\left.\mathrm{O}\right)$ originating from Enceladus' water plume (e.g., Shemansky et al. 1993; Esposito et al. 2005; 
Delamere et al. 2007; Melin et al. 2009; Fleshman et al. 2010; Cassidy and Johnson 2010; Tadokoro et al. 2012). Enceladus supplies $\sim 150-350 \mathrm{~kg} / \mathrm{s}$ of neutral $\mathrm{H}_{2} \mathrm{O}$ gas to the magnetosphere (e.g., Hansen et al. 2006). The neutral cloud is an important loss process of plasmas in the inner magnetosphere (e.g., Paranicas et al. 2007, 2008). Cassini observations suggest that ions with several tens of $\mathrm{keV}$ are significantly lost by charge exchange (e.g., Krimigis et al. 2007; Paranicas et al. 2008). An understanding of the neutral cloud is required to understand plasma dynamics in the magnetosphere. The observations by the Hubble Space Telescope (HST) reveal numerous OH (Shemansky et al. 1993). The observations by the Cassini spacecraft clarify $\mathrm{H}_{2} \mathrm{O}$ plume with temporal variation (e.g., Porco et al. 2006; Spencer et al. 2006; Hansen et al. 2008) and $\mathrm{O}$ with temporal and spatial variations (e.g., Esposito et al. 2005; Melin et al. 2009). Although the O distributions observed by Cassini show asymmetries in the local time distribution, it is difficult to explain the asymmetric distribution, since the orbital period of Enceladus ( $~ 1.4$ days) is shorter than the period of observations ( $\sim$ order of weeks). EXCEED will observe the emission of O I at $1304 \AA$ in the inner magnetosphere within 10 Rs. It is expected to produce a mosaic per 1 day. EXCEED will produce the image at $\mathrm{O}$ I to clarify the local time structure with a temporal variation. The atomic oxygen observation by EXCEED is a key parameter for understanding the neutral and plasma dynamics in Saturn's magnetosphere.

\section{Instrument and Calibrations}

\subsection{EXCEED: Extreme Ultraviolet Spectroscope for Exospheric Dynamics}

The EXCEED instrument has a parabolic off-axis mirror (diameter $20 \mathrm{~cm}, \mathrm{~F}=8$, reflection angle $5.4^{\circ}$ ) that feeds a prime focus spectrograph with a photon counting microchannel plate detector and resistive anode readout (Figs. 3 and 4).

The entrance mirror is made of SiC substrate manufactured by Nicon Co. The chemical vapor deposition of silicon carbide (CVD-SiC) is coated on the surface. Reflectivity calibrated in the EUV spectral range is presented in Fig. 5 together with a theoretical curve.

The movable slit is placed on the focal plane of the mirror (Fig. 6, upper panel). It has three kinds of slit shapes (narrow, wide, and dumbbell) and three kinds of transmittance characteristics (transparent, with a thin film of indium, with a glass of calcium fluoride). Therefore, nine kinds of slits are in a line and are selective by the worm drive.

Calcium fluoride $\left(\mathrm{CaF}_{2}\right)$ bandpass filters are placed on the slits for aversion to our mission full abort. $\mathrm{CaF}_{2}$ has a sharp cutoff of transmittance at the shortward of $1300 \AA$. If we had encountered with high irradiation of stray light (for example, Lyman- $\alpha$ line at $1216 \AA$ ), we must have used the slit with the $\mathrm{CaF}_{2}$ filter at the expense of sensitivity and shortward spectral measurements. Indium (In) has a relatively wide bandpass in 700-1100 A. We may use the In-filter for the observation of the bright EUV stars to avoid the signal saturation at the detector electronics. Transmittances of the materials are shown in Fig. 5.

Behind the slit, a laminar-type grating with a toroidal curvature surface manufactured by Shimazu Co. is placed (the substrate was manufactured by Nikon Co.). It was optically fabricated by a holographic method. The line density is 1800 lines $/ \mathrm{mm}$. The calibrated efficiency at first order is presented in Fig. 5 together with a theoretical curve.

A photon sensor takes a spectral image. We employ five-stage (V-Z stack) microchannel plate (MCP) assembly with a resistive anode position encoder (RAE). This type of detector has already been used in various space missions such as UPI on KAGUYA spacecraft (Murakami et al. 2010), and IMAP on international space (Yoshikawa et al. 2011a). The 


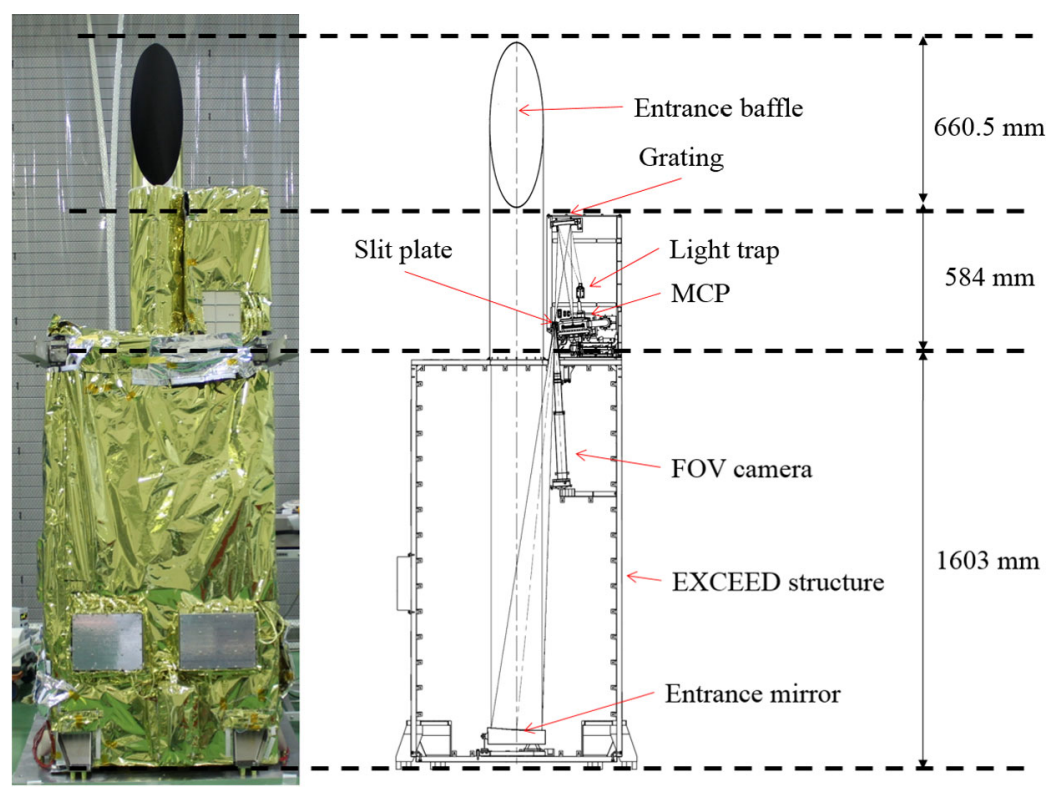

Fig. 3 Photograph (Left) and the optical layout (Right) of EXCEED on Sprint-A. Sharp edge at the top of the entrance baffle (cut angle of $20^{\circ}$ ) defines the lower limit of the observable elongation angle. Incident photons coming from the entrance baffle are collected by the off-axis parabolic mirror with a $20 \mathrm{~cm}$ diameter and focused onto the slit plate. The reflection angle is $5.4^{\circ}$. The field-of-view guiding camera (FOVGC) identifies the position of the target on the slit plate. Behind the slit plate, the diffraction grating and micro channel plate (MCP) assembly are placed. This optical layout realizes that the target radiations (i.e. planetary EUV emissions) can reach the detector with only two reflections. (Modification of Fig. 2 in Yoshioka et al. 2011, 2013)

effective area of the MCP is divided into $1024 \times 1024$ imaginary pixels. The first face of MCP is coated with a photocathode (CsI) to increase the quantum efficiency except around $1216 \AA$ (Lyman- $\alpha)$. The MCP was kept in the vacuumed chamber on board so that CsI should prevent from degradation by moisture in the air before the launch. After the outgassing from the spacecraft dried up, the front door of the vacuum chamber was opened by activating paraffin actuator. The volume expansion due to the solid-to-liquid phase change of paraffin delivers the actuator stroke. A photograph of the detector unit is shown in Fig. 7.

Incident photons initiate cascades of multiple electrons at the photocathode (CsI). The resulting electron cloud strikes the surface of the RAE. The event location $(X, Y)$ on RAE is calculated by manipulating the voltages measured at the four corners. Four charge-amplifiers (A225, manufactured by Amptek Co.) convert the total charge of electrons to the equivalent voltage. While a peak-hold detector (PH300, manufactured by Amptek Co.) keeps the peak voltage in the event, ADC digitized it into 16 bits. Figure 8 is a block diagram of the electronics. Black lines of the upper panel in Fig. 9 show the pulse-height distributions with various levels of HV applied in the ground-based, and the red one is available from in-flight calibration. The absolute sensitivity was calibrated before the launch by using a NIST-calibrated photodiode. Details of the ground-based calibration were given by Yoshioka et al. (2011, 2013).

The spectrograph covering 520-1480 $\AA$ in first order with a dispersion of approximately $1 \AA$ per pixel was built with the above optical system. The data processor has a temporal memory stores 1300 photon events (position and time). Position data consists of four outputs 
Fig. 4 Two photographs of the spectrometer put down sideways. The lower photograph shows the inside of the spectrometer with side panels removed off. Photons incident from the slit strike the grating and are diffracted. The microchannel plate assembly (MCP) takes a spectral image in

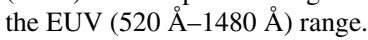
The MCP assembly is kept in vacuum with an $\mathrm{MgF}_{2}$-window closed during the ground-based operations to prevent the moisture. The glass of $\mathrm{MgF}_{2}$ is transparent in the spectral region above $1150 \AA$ A t therefore, even if the window is stalled at closed position in space, the emissions in the spectral region above $1150 \AA$ are observed. The lower photograph is modified from Fig. 3 of Yoshioka et al. (2011, 2013)
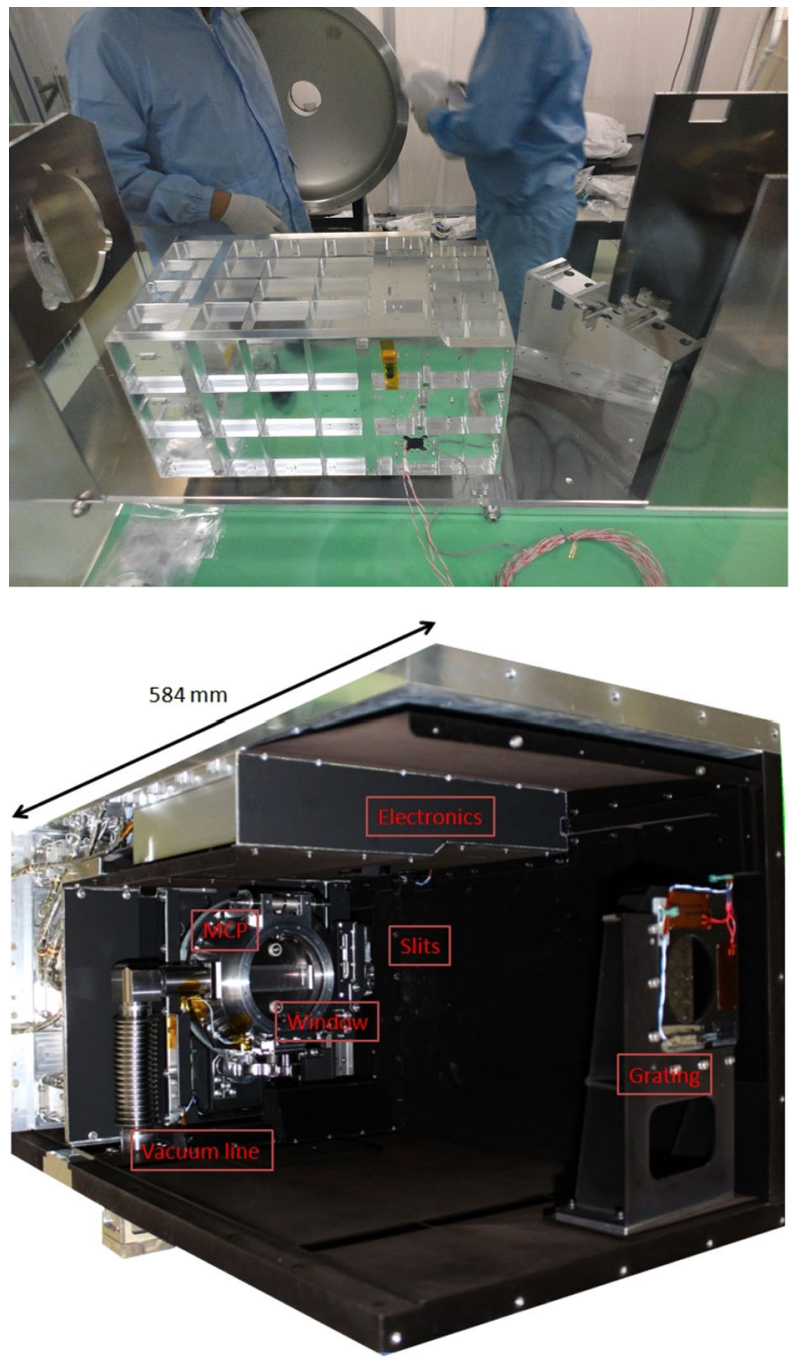

from the channels (A, B, C, D), each is digitized by 16-bit, each channel data is stored with 16-bit. We calculate the position of each photon event by $\mathrm{X}=(\mathrm{A}+\mathrm{B}) /(\mathrm{A}+\mathrm{B}+\mathrm{C}+\mathrm{D})$, $\mathrm{Y}=(\mathrm{A}+\mathrm{C}) /(\mathrm{A}+\mathrm{B}+\mathrm{C}+\mathrm{D})$ and produce the spectral image on the ground. This method has a significant benefit. We can obtain not only a spectral image ( $X-Y$ matrix) but also a pulse-height distribution (PHD) of signals, i.e., histogram of amplitudes of $\mathrm{A}+\mathrm{B}+\mathrm{C}+\mathrm{D}$. It realizes the detector's health check even during the observation.

\subsection{Field-Of-View Guidance Camera (FOVGC)}

The SPRINT-A satellite has a three-axis-stabilized attitude control system, but the original absolute pointing accuracy was poor ( \pm 1.5 arc-minutes $)$. Therefore, the Field-Of-View Guidance Camera (FOVGC) is boarded to improve the pointing accuracy. If the target (planet) image produced by the entrance mirror of EXCEED does not fall onto the slit, the 


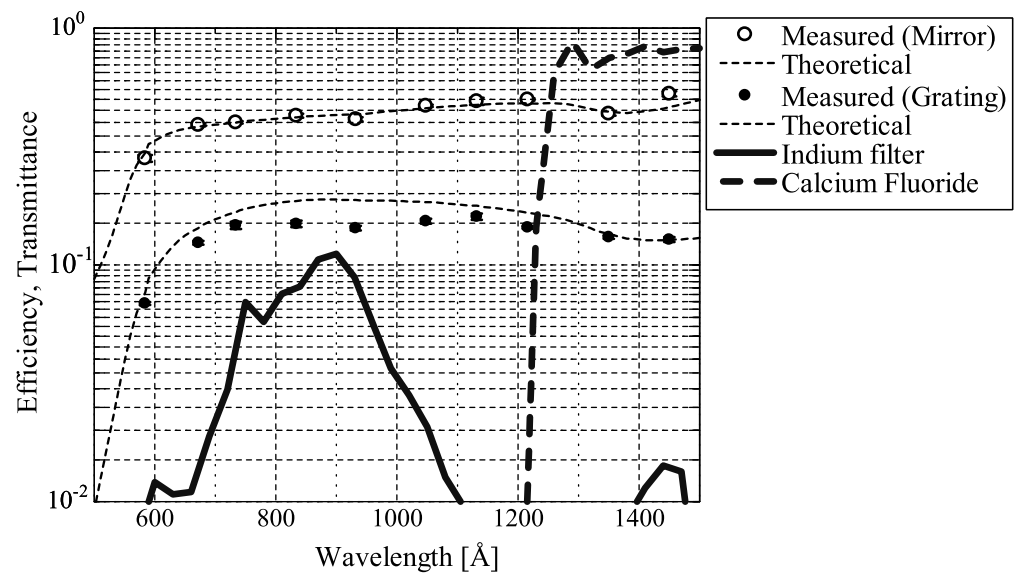

Fig. 5 Characteristics (efficiency and transmittances) of optical components. Measured values and theoretical curves are presented. The entrance mirror, manufactured by Nikon Corporation, has a parabolic shape of surface coated with CVD-SiC (substrate is made of $\mathrm{SiC}$ ) with an effective diameter of $20 \mathrm{~cm}$. The reflectivity was measured at the incident angle of $5.4^{\circ}$. The holographic diffraction grating with a $50-\mathrm{mm}$ effective diameter was manufactured by Shimazu Corporation. The diffraction efficiency at first order was measured at the incident angle of $10.4^{\circ}$

image reflected at the outer slit frame is captured by FOVGC. It has an FOV by $240^{\prime \prime} \times 240^{\prime \prime}$. A mission by data processor (MDP) identifies the centroid of the reflected image and calculates the gap and direction between the slit and target every $5 \mathrm{~s}$. The gap and direction are informed the spacecraft attitude control system (Common Bus system in Sprint series). The satellite bus system leads the spacecraft attitude system so that the line-of-sight (LOS) of EXCEED should direct the target.

Figure 10 schematically shows the dumbbell-shaped slit and target. The FOV of FOVGC is shown as gray rectangles. The sequence from label (1) to (3) is taken to introduce the target onto the slit. When the planet's disk overlaps with the edge of the FOV (as shown in label (1a)), the bus system carries out the attitude maneuver until FOVGC captures a complete disk image with the help of the centroid position calculated by MDP. When the target image falls completely onto the slit (labels (1b)), the maneuver is carried out in the direction perpendicular to the slit until FOVGC captures the disk. Finally, the bus system maneuvers the attitude so as to move the centroid of the target to the uplinked position in order to capture the observation target within the slit (label (2)). After that, EXCEED starts observing the target of interest (e.g., both the IPT and the Jovian aurora). During the observation, the bus system controls the attitude to keep the centroid position of the reference target in FOVGC (star mark in the disk of label (3)). With the help of this sequence, Sprint-A has achieved the pointing accuracy of 2 arc-seconds. The in-flight calibration regarding the pointing stability is described in an accompanying paper (Yamazaki et al. 2014).

\subsection{In-Orbit Calibration: Operational HV Level, Plate-Scale, Spatial/Spectral Resolutions, and Overall Sensitivity}

As cited in Sect. 3.1, we can obtain the pulse-height distribution (PHD) of 5-stage MCP assembly from the raw data of EXCEED. The upper panel of Fig. 9 shows the PHDs with high voltage applied in space (red line) and on the ground-based calibrations (black lines). On the ground, the average gain (peak of the distribution) became higher, growing from 

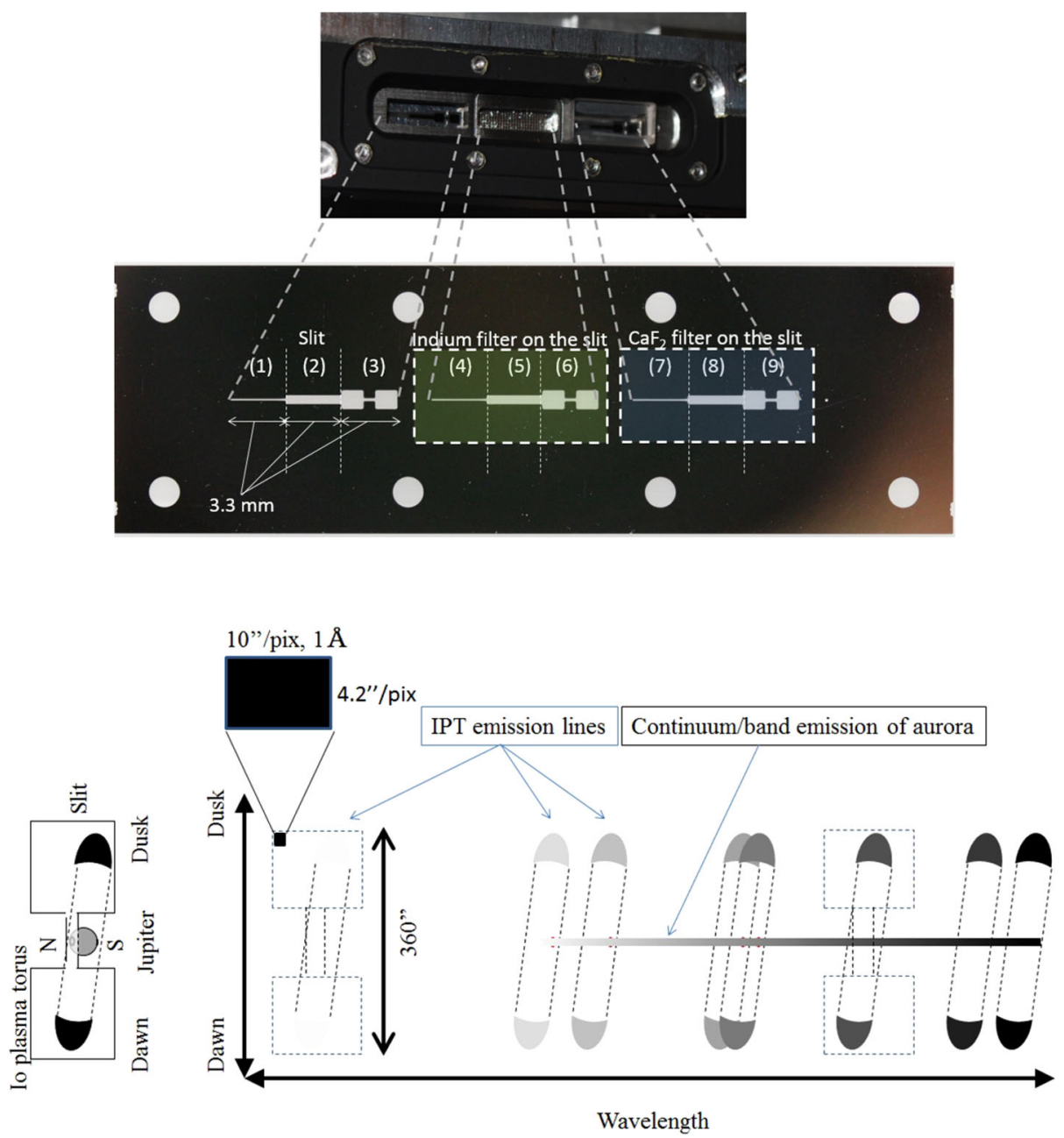

Fig. 6 (Upper panel) a photograph of a slit plate. Three kinds of slit shapes and two bandpass filters are installed. They are selective. The positions at (1), (2), and (3) are narrow, wide, and "dumbbell-shaped" slits, respectively. The positions at (4), (5), and (6) are the same slit pattern as the left, but with an indium filter covered. The slits with the bandpass filter made of calcium fluoride $\left(\mathrm{CaF}_{2}\right)$ are placed at positions of (7), (8), and (9). The narrow slits placed at (1), (4), and (7) have corresponding angular widths of $10^{\prime \prime}$. The three wide slits are $60^{\prime \prime}$. The dumbbell-shaped slits are used to take a spectral image of Jupiter aurora and Io plasma torus simultaneously. All slit lengths are $3.3 \mathrm{~mm}$, which corresponds to an angular width of $360^{\prime \prime}$. The indium filter was manufactured by LUXEL Corporation. The measured transmittances of indium (In) and calcium fluoride $\left(\mathrm{CaF}_{2}\right)$ filters are shown in Fig. 5. (Lower panel) schematic spectral image with the dumbbell-shape slit. The center part of the slit with narrow width will be placed on the polar region of Jupiter, while the two edges are on Io plasma Torus (IPT). Due to the relative motion of IPT, the tilt angle of IPT changes. The spectrograph mixes spatial and spectral information along the $X$-axis. The scale is presented

$1 \mathrm{pC}$ to $4.5 \mathrm{pC}$, while the applied level of high voltage increased from $-3.4 \mathrm{kV}$ to $-3.7 \mathrm{kV}$. However, increment of high voltage in space might induce the discharge and potentially result in a mission failure. In order to avoid the worst case, we have increased the applied voltage step by step for approximately 3 days. As previously stated, we can obtain a pulse- 

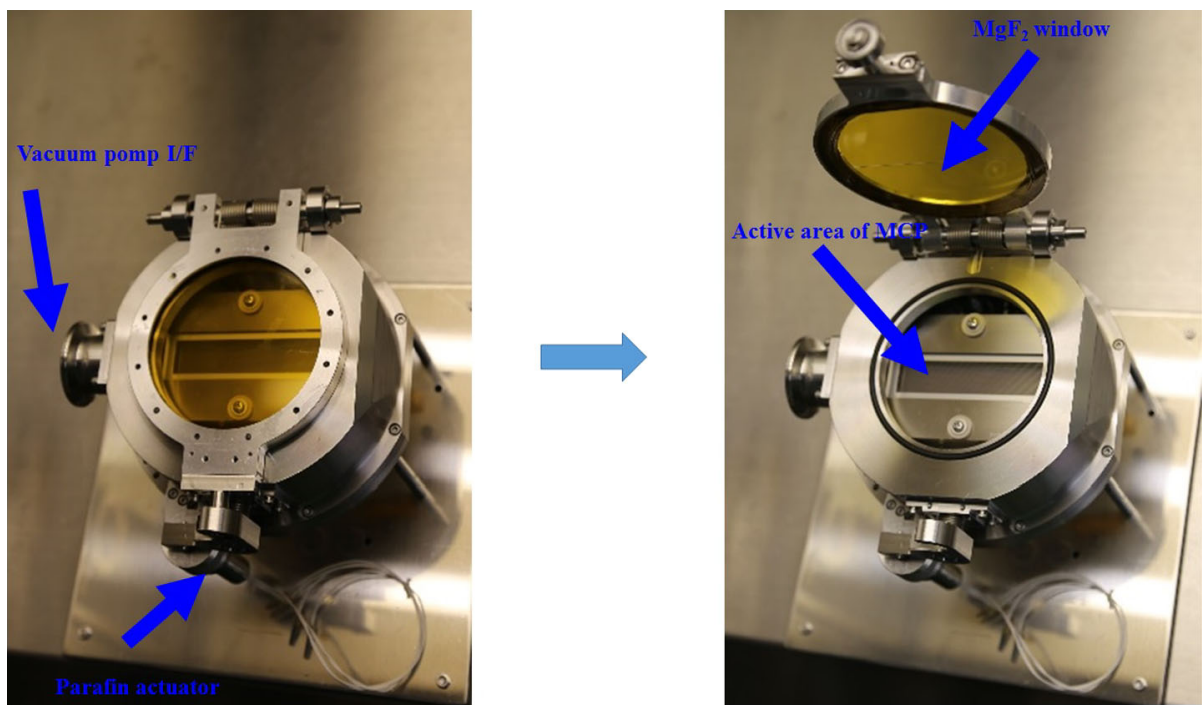

Fig. 7 Photographs of the microchannel plate assembly kept in the space-qualified vacuum chamber. The door with the $\mathrm{MgF}_{2}$ window was open by the paraffin actuator. Cesium iodide (CsI) was deposited on the first face of the MCP assembly as photoelectric material to increase the quantum efficiency. Photographs were taken before deposition of CsI

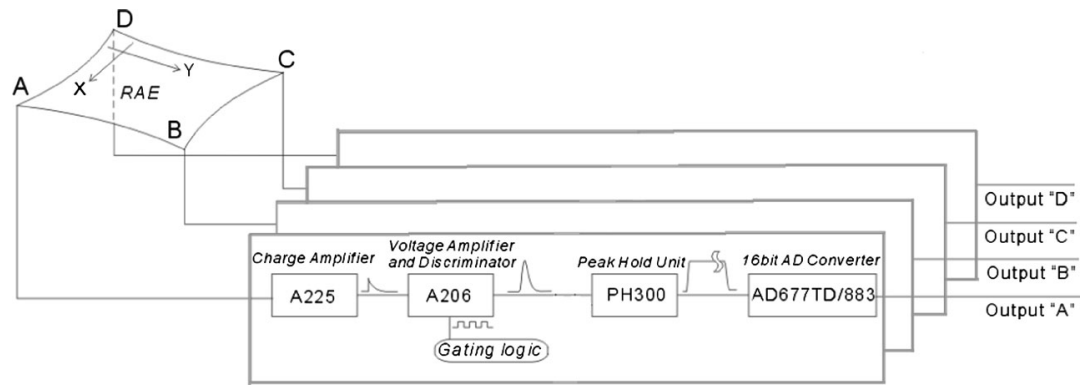

Fig. 8 Block diagram of the electronics for the position determination. Amplifiers, pulse-shapers with discriminators, peak-hold components are manufactured by AMPTEK Co. Four digitized signals, (A)-(D), are sent to the ground every photon event. On the ground, we can determine the position $(X, Y)$ by using the signals

height distribution and spectral image simultaneously. The lower panels of Fig. 9 show the PHDs available at $584 \AA$ (labeled as (a)), $834 \AA$ as (b), $1216 \AA$ as (c), and $1304 \AA$ as (d) for the first light of the geocorona. Their features of PHDs were similar; however, you can see the peak of the gain is lower, while the wavelength is longer (energy is lower). The wavelength dependence is by the nature of the detector due to the interaction between a pore of MCP and incident photons. We found that the minimum level of $\mathrm{HV}$ was $-3.5 \mathrm{kV}$ and that it realized fairly good spatial resolution of the detector at the longer wavelength. The slight difference in the peak of PHD between the ground and in-flight calibrations at $-3.5 \mathrm{kV}$ might be due to the degradation of MCP gain after the launch. 


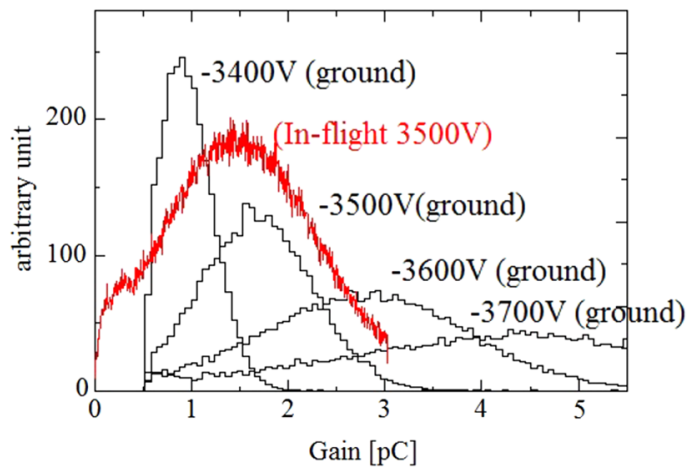

(a) 20131119, wavelength $=584[\mathrm{~A}]$

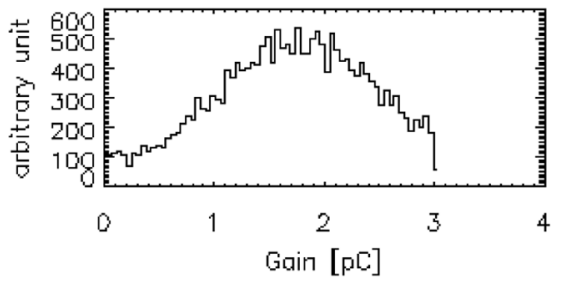

(c) 20131119 , wavelength $=1216[\mathrm{~A}]$

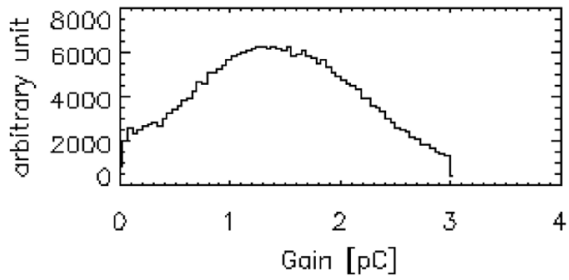

(b) 20131119, wavelength $=834$ [A]

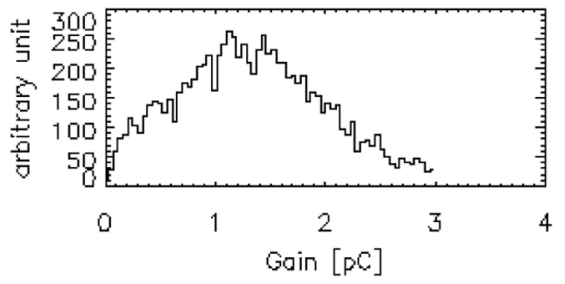

(d) 20131119 , wavelength $=1304[\mathrm{~A}]$

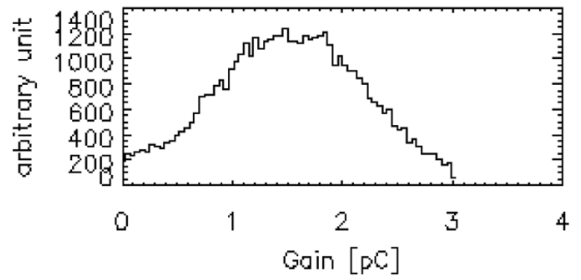

Fig. 9 (Upper) Pulse-height distributions (PHDs) of the five-stage micro channel plate assembly. Black lines show the PHDs available from ground-based calibration, and the red one is from in-flight calibration. (Lower) PHDs at geocoronal wavelengths

Overall sensitivity was calibrated by a star (GD71) observation. GD71 (WD0459+ 158), first cataloged by Giclas et al. (1965), is a bright nearby hot DA white dwarf in the middle of the aforementioned temperature range $(T=32000 \mathrm{~K})$. It has been studied extensively at optical, UV, EUV and near-IR spectral range and is widely used as a photometric and spectrophotometric calibration star (Dobbie et al. 2002). The calibrated sensitivity curve versus wavelength is shown in Fig. 11. Below $930 \AA$, we normalized the sensitivity curve obtained at the ground-based calibration before the launch to fit the curve at longer wavelength, while above $930 \AA$ the overall sensitivity was calibrated in comparison with the model flux of GD71 CALSPEC cataloged. EXCEED has an effective area of more than $1 \mathrm{~cm}^{2}$ in the spectral range of 580-1350 ̊ except near $1216 \AA$, where the strong geocorona emission (Lyman- $\alpha$ ) illuminates; therefore we intended to reduce the sensitivity. We will have an opportunity to calibrate the sensitivity once a month and to extend the spectral range to shortward.

Using the opportunity of star observation, we have determined the spatial resolution (point-spread function) by 17 arc-seconds (FWHM), the plate scale by 4.2 arc-second/pixel 
Fig. 10 Schematic pictures showing the dumbbell-shaped slit relative to the target (Jovian disk and Io Plasma Torus). Labels (1) to (3) in the upper and lower panels show the sequence of capturing an observation target into the slit. Modification of Fig. 1 of Tsuchiya et al. (2011) (a)

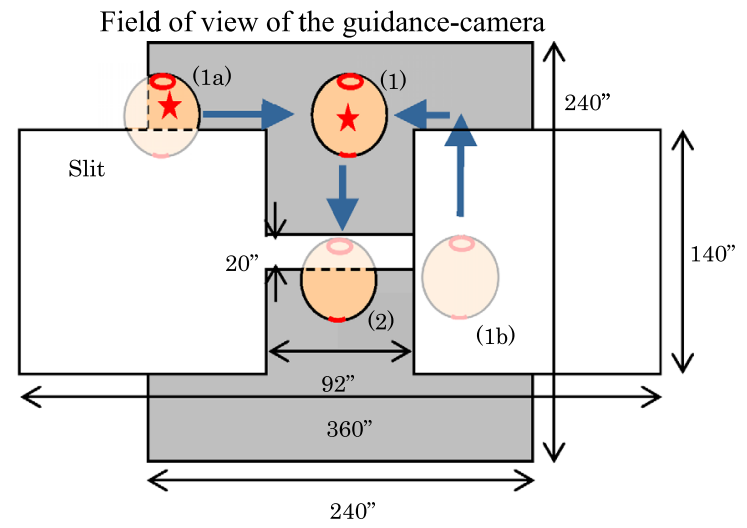

(b)

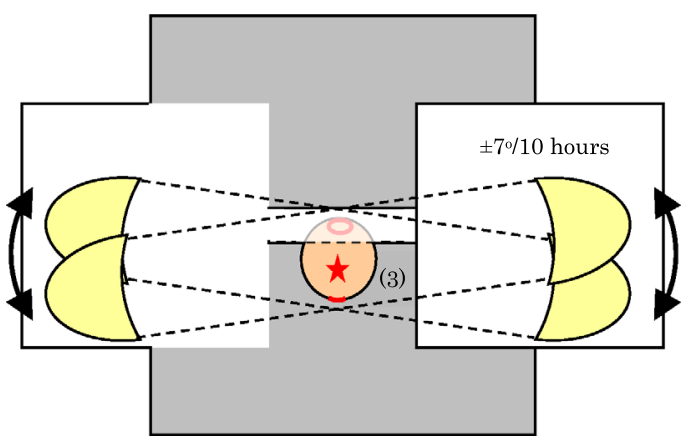

Effective area of EXCEED (in-orbit cal)

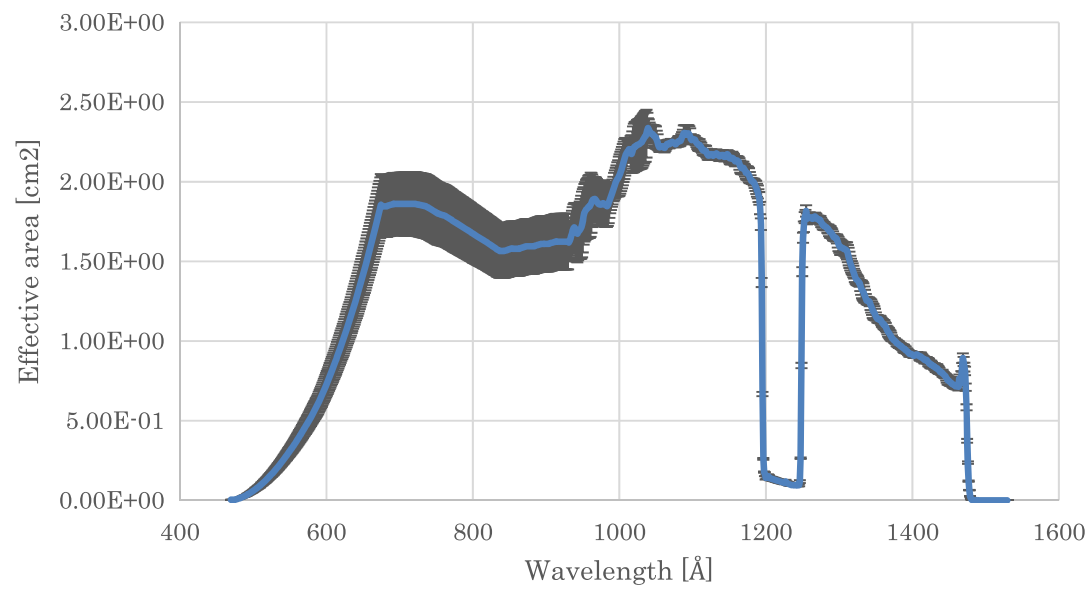

Fig. 11 The overall sensitivity was calibrated by a star (GD 71) observation. GD 71 is the standard UV star for the calibration. Below $930 \AA$, we employed and modified the sensitivity curve obtained at the ground-based calibration before the launch, while above $930 \AA$ the overall sensitivity was calibrated in space using GD71

along the spatial direction, and the FOV in spatial direction by 360 arc-seconds. The spectral resolution is determined by the point-spread function as $3 \AA$ (FWHM). All the dimensions are shown in the lower panel of Fig. 6. 


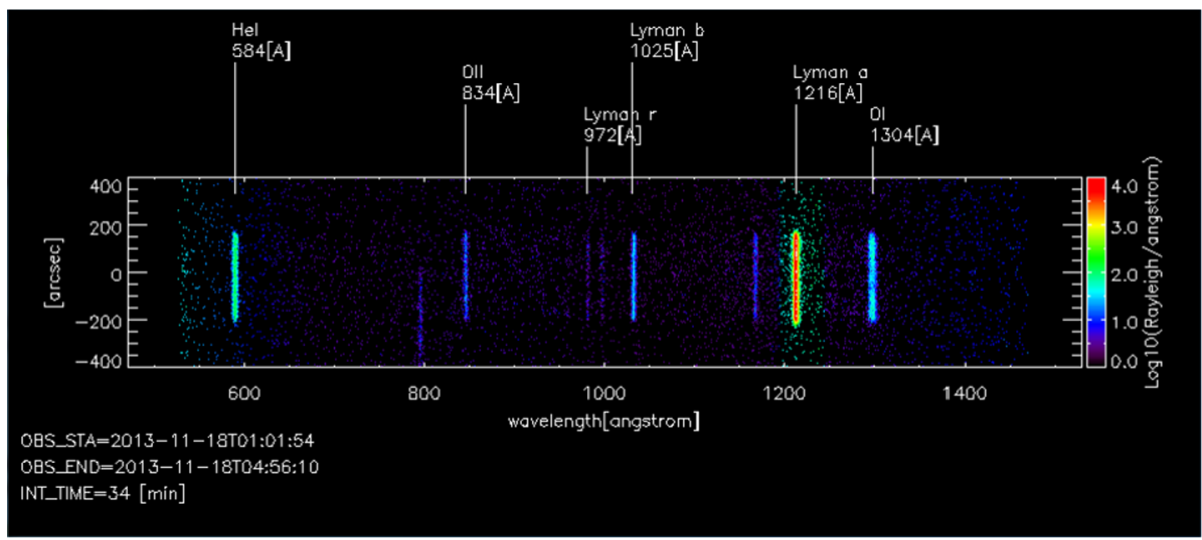

Fig. 12 First data just after the high voltage was applied on MCP by $3.55 \mathrm{kV}$. Geocoronal emissions, Lyman- $\alpha$ at $1216 \AA$, Lyman- $\beta$ at $1025 \AA$, He I at $584 \AA$, O II at $834 \AA$, and O I at $1304 \AA$, are clearly identified. A narcissistic ghost of Lyman- $\alpha$ line appears near $800 \AA$, which was a result of incidence on the face of the detector (MCP), back onto the detector by the grating in zero order. The second order of He I (584 ̊) appeared at the exact position

We have re-considered the necessary exposure periods for visualizing very dark targets (the escaping ions) based on the results from in-flight calibration (effective area versus wavelength, detector noise due to high-energy particle bombardment $\left.\left(2.0 \mathrm{cps} / \mathrm{cm}^{2}\right)\right)$. The details are given in the Appendix. We should integrate the spectra for approximately 30 passes ( $<3$ days) for identification of C II (1335 $\AA$ ) at ionospheric tail and for approximately 120 passes ( $<12$ days) for identification of N II (1085 $\AA$ ). We have scheduled the observation period of Venus from March to May in 2014.

\section{Launch and Orbit}

The epsilon rocket was used to launch a three-axis stabilized satellite (Sprint-A) with EXCEED, making its first flight. The four-stage Epsilon rocket was launched from the Uchinoura Space Center at 05:00 UTC on 14 September 2013. Following its successful insertion into the orbit and deployment of its solar arrays, Sprint-A was renamed Hisaki. The perigee and apogee are $954.05 \mathrm{~km}$ and $1156.87 \mathrm{~km}$, respectively, as planned. The orbital inclination is $29.704^{\circ}$. The orbital period is 104 minutes.

\subsection{Foreground Emissions: Geocorona Measurement}

Before starting the nominal (target-pointing) observation, we had a spectral image of foreground emissions, the so-called geocorona. At the initial phase of the mission, we plan to measure the foreground emissions every three orbit. The look direction is 5 arc-minutes off from the target. Modeling the geocorona brightness is an important skill to increase the possibility to find a rapid enhance of aurora and torus brightness and quantify the amount of Venusian/Martian atmospheres escaping to space. Figure 12 shows the spectra obtained at 01:01:54 UT on November 18, 2013 at the latitude of $21.3^{\circ}$ and the longitude of $136^{\circ}$ at the altitude of $1151 \mathrm{~km}$. Lyman- $\alpha$ at $1216 \AA$, Lyman- $\beta$ at $1025 \AA$, O II at $834 \AA$, O I at $1304 \AA$, and He I at $584 \AA$ are clearly identified with the flat intensity over the slit. Their average intensities for $34-\mathrm{min}$ are $370 \mathrm{R}$ at He I (584 $\AA$ ), $35 \mathrm{R}$ at O II (834 $\AA$ ), $67 \mathrm{R}$ at Lyman- $\beta$ 


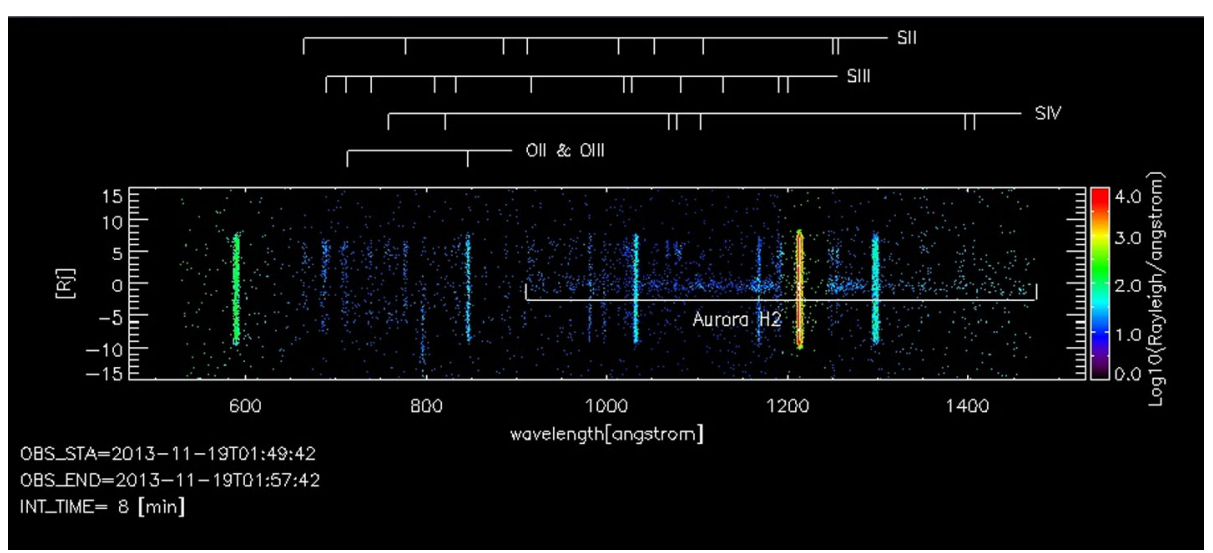

Fig. 13 EUV spectral image of the Io plasma torus and the northern pole of Jupiter with the narrow slit. This spectrum is created by 9-min exposure obtained at beginning at 01:48 UT September 19, 2013, while EXCEED was orbiting at $1150 \mathrm{~km}$ altitude. Foreground emissions had flat intensities along the slit

(1025 ̊), $22 \mathrm{kR}$ at Lyman- $\alpha$ (1216 $\AA$ ), and $143 \mathrm{R}$ at O I (1304 $\AA$ ) and consistent with our present knowledge based on past numbers of observations (e.g., Meier 1991). (These intensities were calibrated based on the stellar calibrations as cited in Sect. 3.3.)

Although contaminations from stray lights and high-energy particles were negligible, a narcissistic ghost line appeared near $800 \AA$ (McCandliss et al. 1998). Bright emission at Lyman- $\alpha(1216 \AA)$ was incident on the face of the detector (MCP), back onto the detector by the grating in zero order. We always have to always be careful about narcissistic ghosts for the identifications of weak emissions. The level of contamination is $1 / 1000$. We will be able to re-examine this feature by the EUV star observations in the near future.

\subsection{First Images of Jupiter and Venus}

The EXCEED observations of Jupiter and Venus represent the highest quality spectra, in terms of spectral/spatial resolutions and instrument sensitivity. The present discussion will focus on the identification of spectral features and comparison with previous observations. Future works will concern the modeling of these spectra in terms of current atmospheric models based on in situ measurements (GALILEO, Venus Express, etc.).

At the time of observation start (November 19, 01:48:42UT), Jupiter was at a solar elongation of $125^{\circ}$ and apparent diameter was 43.6 arc-seconds. The spacecraft was at the altitude of $1150 \mathrm{~km}$ and at the local time of $10.3 \mathrm{~h}$. We placed the narrow slit (in the later half of the observation, we used the dumbbell-shape slit) on Jupiter and applied HV with $-3.5 \mathrm{kV}$ to the detector. Spectral images of the Io plasma torus were obtained every 60 -s. In this study, 9-min exposure (nine spectral images were accumulated) is used in order to improve the signal-to-noise ratio. Figures 13 and 14 are the first spectra of Jupiter by EXCEED. The intensities in the two spectra are calibrated after star observation shown later. Figure 13 shows the highest-spectral resolution image using the narrow slit, while Fig. 14 shows the spectra by the dumbbell-shaped slit. The spacecraft orientation was not controlled perfectly at this stage; therefore, Jupiter was not guided at the exact center of the slit. The right panel of Fig. 14 presents the image from FOVGC. Jupiter was at the edge of the slit. As shown in the lower panel of Fig. 6, this spectrograph mixes spatial and spectral information. The continuum aurora emission appeared at a slightly lower position of the slit than that in Fig. 13, 

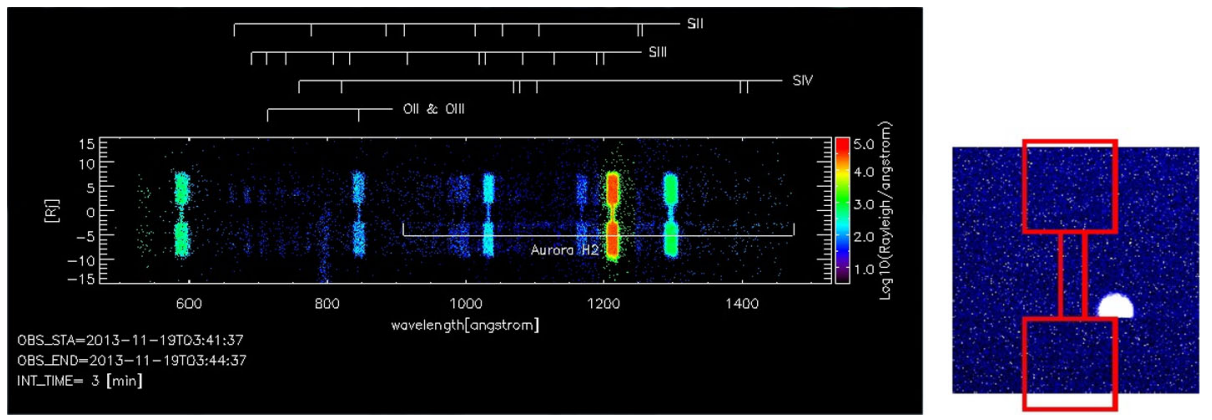

(upper)
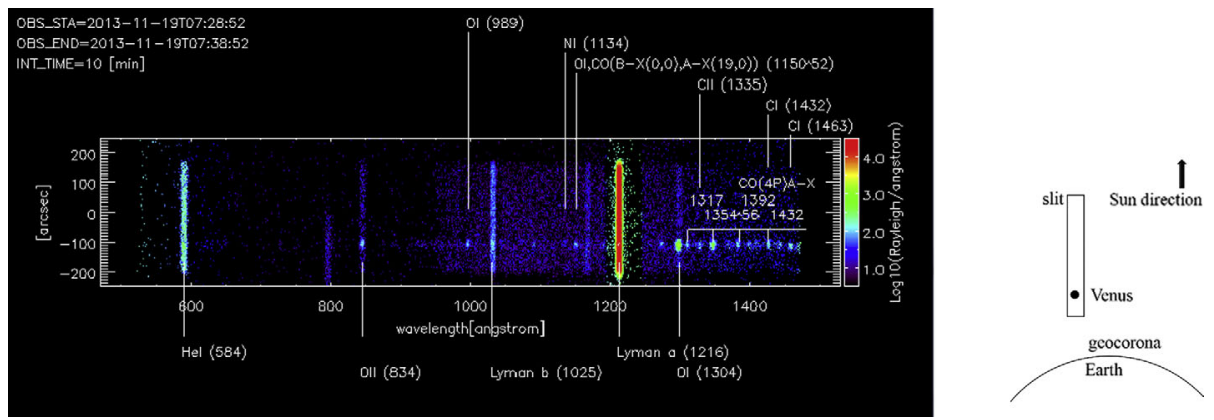

(Lower)

Fig. 14 (Left) spectral image with the dumbbell-shape slit. (Right) image of FOVGC during the exposure. The dumbbell-shape is indicated as red lines. Spacecraft orientation during this period was not controlled yet at the first day, therefore Jupiter was not at the center part of the slit. Emissions of IPT and aurora were observed

emissions from sulfur ions were identified at the slightly shifted due to the finite size of the target.

Both figures show the existence of sulfur ion emissions, e.g. at $680 \AA$ (S III), $765 \AA$ (S II). (For reference, major transitions are indicated at the top.) This spectral image involves at least 11 individual spectral transitions (S II, S III, S IV and O II transitions). We can identify aurorally excited $\mathrm{H}_{2}$ Lyman and Werner bands extending toward shorter wavelength from Lyman- $\alpha$. The S III line at $680 \AA$ is by far the most energetic feature in the EUV spectrum of the torus. The general shape of the profile observed by EXCEED is fairly similar to the UVIS profile by the Cassini spacecraft (Steffl et al. 2004a, 2004b); however, our spectral image seems thinly. The weak emissions, e.g. S II (642 A), need a longer exposure period to appear in the spectrum.

On the same day, Venus was at the elongation angle of $46^{\circ}$ and the apparent diameter was 31.4 arc-seconds. Just after the performances of FOVGC and attitude control system were confirmed, we decided to observe Venus with a wider (60 arc-seconds) slit. The slit was placed along the equator and the Venus disk fell onto the slit completely. This observation started at 07:27 UT. While the spacecraft was at the longitude of $114^{\circ}$, the latitude of $24.9^{\circ}$, a local time of $15.3 \mathrm{~h}$, and the altitude of $1096 \mathrm{~km}$, the first spectral image of Venus was available (Fig. 15).

The spectral image includes the desired disk emissions at Venus as well as contributions from the terrestrial foreground emissions, even though the exposure was limited by 
Fig. 15 (Upper panel) spectral image of Venus using the wide $\left(60^{\prime \prime}\right)$-width slit. The Venus disk image fell onto the slit completely. A large number of individual bands of the carbon monoxide, fourth positive system $\left(\mathrm{A}^{1} \Pi-\mathrm{X}^{1} \Sigma^{+}\right)$, and Hopfield-Birge band $\left(\mathrm{B}^{1} \Pi-\mathrm{X}^{1} \Sigma^{+}\right)$are identified. (Lower panel) The spectrum of the Venus disk in the upper panel

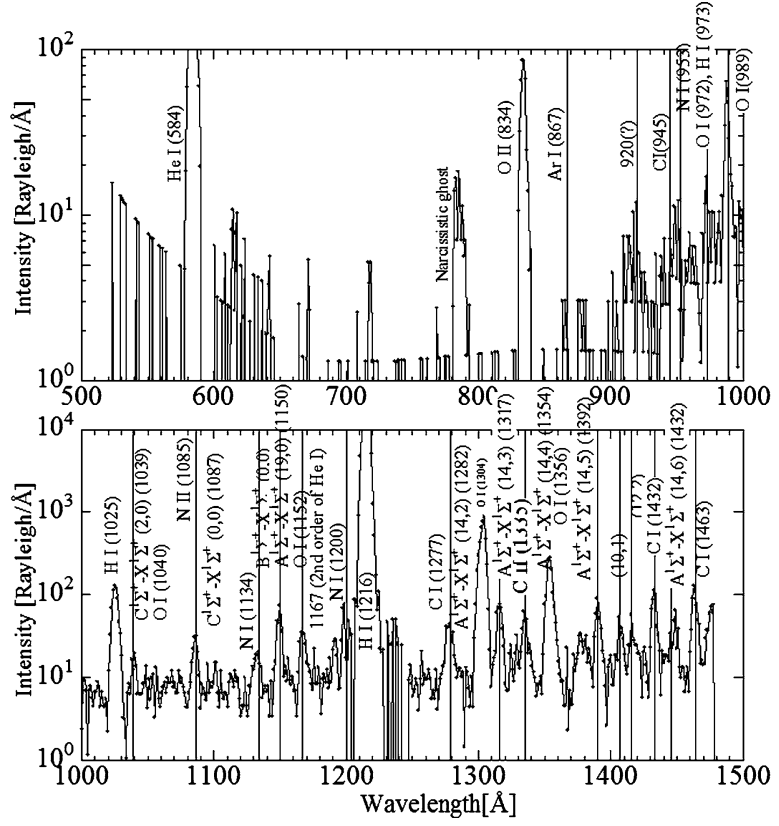

8-minute. Geocoronal emissions illuminated the whole of the slit as shown in the upper panel of Fig. 15, however, the brightness at O II (834 ̊) and O I (1304 ^) around Venus surpassed the geocorona brightness alone. The lower panel of Fig. 15 is the spectrum at the Venus disk. (Cross-sectional view at the Venus disk position of the spectral image.) A large number of individual bands of the CO Fourth Positive $\left(\mathrm{A}^{1} \Pi-\mathrm{X}^{1} \Sigma^{+}\right)$system and HopfieldBirge bands $\left(\mathrm{B}^{1} \Pi-\mathrm{X}^{1} \Sigma^{+}\right)$were seen, however, some were blended with another line. The $4 \mathrm{P}$ bands at $(14,3)-(14,6)$ are due to the optical pumping up by solar Lyman- $\alpha$ line producing the $\mathrm{CO} 4 \mathrm{P}\left(14, v^{\prime \prime}\right)$ band system through resonance with the $(14,0)$ band (Derrance 1981). Cascading $4 \mathrm{P}$ products at $(10,0)$ and $(12,2)$ were identified on the disk spectra in addition to the principal C II multiplets (1335 $\mathrm{A})$ and O I (989 ̊), whose existences were previously reported by Feldman et al. (2000) and Hubert et al. (2012). The intensities of C II (1335 $\AA$ ) and of O I (989 A) were approximately $100 \mathrm{R}$, which are consistent with the past measurements.

The emission at $867 \AA$ is probably ascribed to Ar I, that was as previously reported by Stern et al. (1996). However, they did not regard it as Ar I emission, because their measured intensity was approximately two orders of magnitude brighter than expected for Ar I emission. We have measured the intensity at $867 \AA$ by less than $10 \mathrm{R}$, while it was reported as more than $100 \mathrm{R}$ (Stern et al. 1996). Noble gases are highly useful tool for studies of the origin and evolution of planetary atmospheres. We will clarify the existence of Ar I emission $(867 \AA)$ and its intensity with higher S/N ratio after a longer exposure period is taken.

As shown in Sect. 2.2, the feature of escaping ions can be visualized after the accumulation of many spectra available for several days.

\subsection{Data Downlink, Processing, and Distribution of EXCEED}

The on-going nominal operation from January 1st 2014 is shown in Fig. 16. The $X$-axis is time, the $Y$-axis presents the operation mode (highest: observation of a planet, middle: 


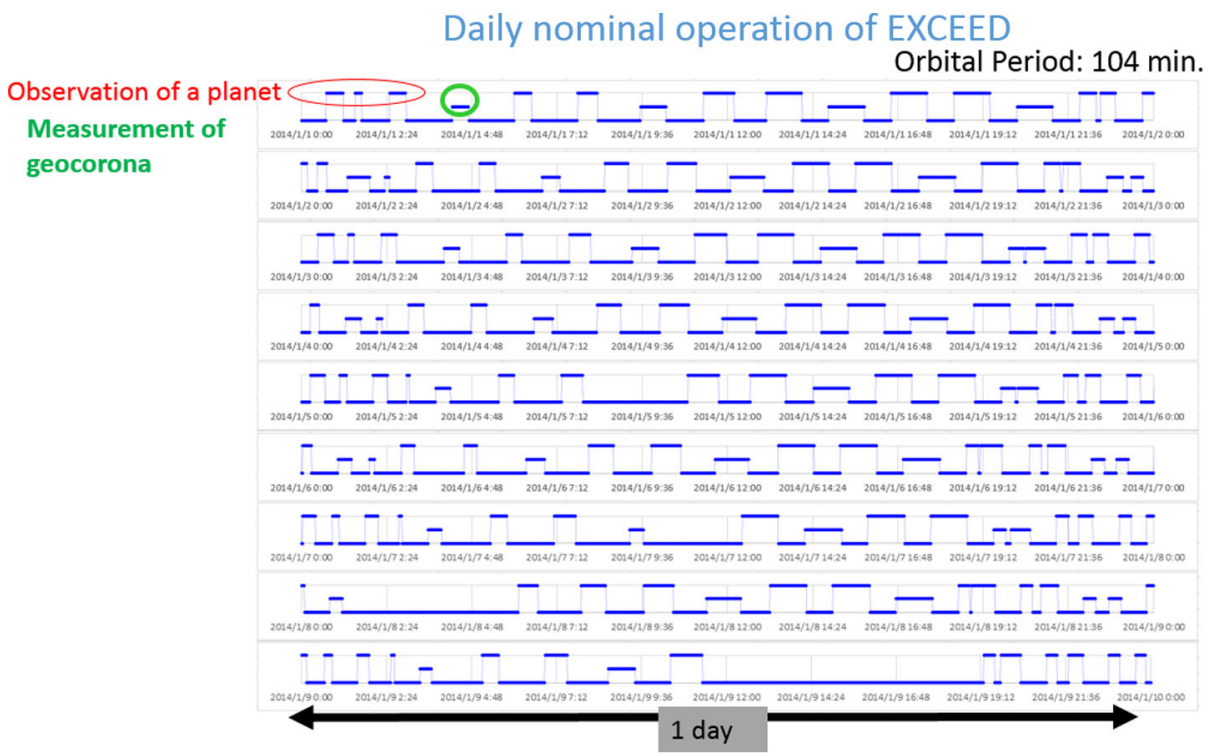

Fig. 16 On-going nominal operation from January 1st 2014. The $X$-axis is time, one horizontal column corresponds to 1-day. $Y$-axis presents the operation mode (highest: observation of a planet, middle: geocorona measurement, lowest: instrument off). EXCEED measures the foreground emissions once a three-orbit. EXCEED has intermittent opportunities to observe a planet around 13 times per day

geocorona measurement, lowest: turning off of the instrument). EXCEED has intermittent opportunities to observe a planet around 13 times per day. EXCEED measures the foreground emissions once in three orbits.

An operation program is uploaded every week. The raw data produced by EXCEED during the observation consist of time-tag photon events, i.e., four 16-bit digitized channel outputs with time code for each photon. The data table is produced every minute. Science, engineering, and housekeeping data are stored on board the spacecraft and downlinked to JAXA ground network. Raw data is converted to spectral images (matrix: $1024 \times 1024$ : LEVEL-1 data). Some corrections are made and additional information is attached to convert it to LEVEL-2 data.

The LEVEL-2 data will be available to the space science community and general public after the examination by the EXCEED team. The LEVEL-2 data are accessible on the Web for display and download from the Center for Science satellite Operation and Data Archive (C-SODA) in ISAS/JAXA. Documentation and analysis software will also be downloaded.

Acknowledgments The authors thank the operation and project members of Sprint-A for their support. The major mechanical components were fabricated by Sumitomo Heavy Industries, Ltd. The microchannel plates were provided by Hamamatsu Photonics, the metallic filter was provided by Luxel Corporation, the entrance mirror was provided by Nikon Corporation and the diffractive grating was provided by Shimazu Corporation.

Open Access This article is distributed under the terms of the Creative Commons Attribution License which permits any use, distribution, and reproduction in any medium, provided the original author(s) and the source are credited. 
Fig. 17 The $X$-axis presents the numbers of passes for the observation necessary for signal-to-noise ratio $(S / \sqrt{N})$ equals one. The $Y$-axis is the intensity in the unit of Rayleigh. Horizontal lines are the expected intensities (see the Appendix)

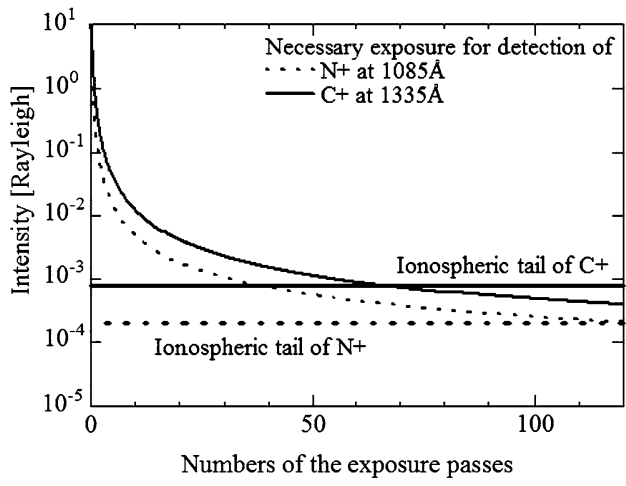

\section{Appendix: Estimate of Intensity of Venus Ionospheric Tail at C II (1335 ̊) and N II (1085 ̊̊)}

Our initial study of the necessary exposure period was presented by Yoshikawa et al. (2011b). A previous study dealt with Venusian O II (834 $\AA$ ) emission in the ionospheric tail, i.e., we investigated the feasibility to identify escaping oxygen ions to space at this wavelength. We concluded that $100-\mathrm{ks}$ exposure was necessary. This estimate is still valid with minor modifications.

Here, we study the feasibility of detection at C II (1335 $\AA)$ and N II (1085 $\AA$ ) in the ionospheric tail, based on the in-flight calibration result. The estimation procedure is the same as the previous study.

In Fig. 17, the $X$-axis is the necessary number of passes so that the signal-to-noise ratio $(S / \sqrt{N})$ equals 1 .

We applied the result by Fox and Sung (2001), who presented the ionospheric density $\left(\mathrm{O}^{+}, \mathrm{C}^{+}, \mathrm{N}^{+}\right.$, etc. $)$versus the altitude. We applied the density ratio at the altitude of $300 \mathrm{~km}$ from their result, and assume (1) the escaping flux of $\mathrm{O}^{+}$to be $5 \times 10^{25} \mathrm{~s}^{-1}$ and (2) $\mathrm{C}^{+}$and $\mathrm{N}^{+}$escaping fluxes to be proportional to the density ratio at $300 \mathrm{~km}$. Then we calculated the column density of $\mathrm{C}^{+}$and $\mathrm{N}^{+}$across the ionospheric tail at the distance of $11 \mathrm{Rv}$ from the planet center (Rv: radius of Venus). The ionospheric tail is assumed to have a cylindrical shape with $6 \mathrm{Rv}$ diameter and filled with escaping fluxes of $\mathrm{C}^{+}$and $\mathrm{N}^{+}$ions. The antisunward flow velocity is assumed to be $400 \mathrm{~km} / \mathrm{s}$. The resultant column densities of $\mathrm{C}^{+}$and $\mathrm{N}^{+}$are $3 \times 10^{7}$ and $2 \times 10^{7}$ ions $/ \mathrm{cm}^{2}$, respectively.

When the g-factors (emission rate factors) are assumed to be $2.8 \times 10^{-5} \mathrm{~s}^{-1}$ (C II: $1335 \AA$ ) and $9.7 \times 10^{-6} \mathrm{~s}^{-1}$ (N II: $1085 \AA$ ) at Venus, the intensities of $0.8 \mathrm{mR}$ and $0.2 \mathrm{mR}$ are expected, respectively. Figure 17 suggests that the necessary exposure periods (the numbers of an observation pass) are approximately 30 passes ( $<3$ days) for C II (1335 $\AA$ ) and 120 passes ( $<10$ days) for N II (1085 $⿱$ ) .

\section{References}

F. Bagemal, Plasma conditions inside Io's orbit: Voyager measurements. J. Geophys. Res. 90(A1), 311-324 (1985)

T.A. Bida, R.M. Killen, T.H. Morgan, Discovery of calcium in Mercury's atmosphere. Nature 404, 159-161 (2000)

A.L. Broadfoot, B.R. Sandel, D.E. Shemansky, J.C. McConnell, G.R. Smith, J.B. Holberg, S.K. Atreya, T.M. Donahue, D.F. Strobel, J.L. Bertaux, Overview of the Voyager ultraviolet spectrometry results through Jupiter encounter. J. Geophys. Res. 86(A10), 8259-8284 (1981) 
J.L. Burch, J. Goldstein, W.S. Lewis, D.T. Young, A.J. Coates, M.K. Dougherty, N. André, Tethys and Dione as sources of outward-flowing plasma in Saturn's magnetosphere. Nature 447, 833-835 (2007)

T.A. Cassidy, R.E. Johnson, Collisional spreading of Enceladus' neutral cloud. Icarus 209, 696-703 (2010)

E. Chassefière, J.-L. Maria, J.-P. Goutail, E. Quémerais, F. Leblanc, S. Okano, I. Yoshikawa, O. Korablev, V. Gnedykh, G. Naletto, P. Nicolosi, M.-G. Pelizzo, J.-J. Correia, S. Gallet, C. Hourtoule, P.-O. Mine, C. Montaron, N. Rouanet, J.-B. Rigal, G. Muramaki, K. Yoshioka, O. Kozlov, V. Kottsov, P. Moisseev, N. Semena, J.-L. Bertaux, M.-T. Capria, J. Clarke, G. Cremonese, D. Delcourt, A. Doressoundiram, S. Erard, R. Gladstone, M. Grande, D. Hunten, W. Ip, V. Izmodenov, A. Jambon, R. Johnson, E. Kallio, R. Killen, R. Lallement, J. Luhmann, M. Mendillo, A. Milillo, H. Palme, A. Potter, S. Sasaki, D. Slater, A. Sprague, A. Stern, N. Yan, PHEBUS: a double ultraviolet spectrometer to observe Mercury's exosphere. Planet. Space Sci. 58, 201-223 (2010)

P.A. Delamere, F. Bagenal, V. Dols, L.C. Ray, Saturn's neutral torus versus Jupiter's plasma torus. Geophys. Res. Lett. 34, L09195 (2007)

S.T. Derrance, The carbon monoxide fourth positive bands in Venus dayglow 1. Synthetic spectra. J. Geophys. Res. 86(A11), 9115-9124 (1981)

P.D. Dobbie, F. Kenyon, R.F. Jameson, S.T. Hodgkin, Far-red optical colours of late-M and L dwarfs. Mon. Not. R. Astron. Soc. 331, 445-452 (2002)

L.W. Esposito, J.E. Colwell, K. Larsen, W.E. McClintock, A.I.F. Stewart, J.T. Hallett, D.E. Shemansky, J.M. Ajello, C.J. Hansen, A.R. Hendrix, R.A. West, H.U. Keller, A. Korth, W.R. Pryor, R. Reulke, Y.L. Yung, Ultraviolet imaging spectroscopy shows an active Saturnian system. Science 307, 12511255 (2005)

P.D. Feldman, E.B. Burgh, S.T. Durrance, A.F. Davidsen, Far-ultraviolet spectroscopy of Venus and Mars at $4 \AA$ A resolution with the Hopkins ultraviolet telescope on Astro-2. Astrophys. J. 538, 395-400 (2000)

B.L. Fleshman, P.A. Delamere, F. Bagenal, A sensitivity study of the Enceladus torus. J. Geophys. Res. 115, E04007 (2010)

J.L. Fox, K.Y. Sung, Solar activity variations of the Venus thermosphere/ionosphere. J. Geophys. Res. 106, 21305-21335 (2001)

L.A. Frank, W.R. Paterson, Observations of plasmas in the Io torus with the Galileo spacecraft. J. Geophys. Res. 105(A7), 16017-16034 (2000)

S. Fukuda, S. Sawai, S. Sakai, H. Saito, T. Nakagawa, T. Tohma, J. Takahashi, K. Kitade, Flexible standard bus for ISAS/JAXA small scientific satellite series, in 26th International Symposium on Space Technology and Science, ISTS, Hamamatsu, Japan, 2008-f-17 (2008)

H.L. Giclas, R. Burnham, N.G. Thomas, A List of White Dwarf Suspects I: Special Objects of Small Proper Motion from the Lowell Survey, vol. 6 (Lowell Observatory, Flagstaff, 1965), pp. 155-164

C.J. Hansen, L.W. Esposito, A.I.F. Stewart, J. Colwell, A. Hendrix, W. Pryor, D. Shemansky, R. West, Enceladus' water vapor plume. Science 311, 1422-1425 (2006)

C.J. Hansen, L.W. Esposito, A.I.F. Stewart, B. Meinke, B. Wallis, J.E. Colwell, A.R. Hendrix, K. Larsen, W. Pryor, F. Tian, Water vapour jets inside the plume of gas leaving Enceladus. Nature 456, 477-479 (2008)

T.W. Hill, A.M. Rymer, J.L. Burch, F.J. Crary, D.T. Young, M.F. Thomsen, D. Delapp, N. Andre, A.J. Coates, G.R. Lewis, Evidence for rotationally driven plasma transport in Saturn's magnetosphere. Geophys. Res. Lett. 32, L14S10 (2005)

B. Hubert, J.-C. Gérard, J. Gustin, D.V. Bisikalo, V.I. Shematovich, G.R. Gladstone, Cassini-UVIS observation of dayglow FUV emissions of carbon in the thermosphere of Venus. Icarus 220, 635-646 (2012)

S. Kameda, I. Yoshikawa, J. Ono, H. Nozawa, Time variation in exospheric sodium density on Mercury. Planet. Space Sci. 55, 1509-1517 (2007)

S.M. Krimigis, N. Sergis, D.G. Mitchell, D.C. Hamilton, N. Krupp, A dynamic, rotating ring current around Saturn. Nature 450, 1050-1053 (2007)

W.S. Kurth, D.A. Gurnett, J.T. Clarke, P. Zarka, M.D. Desch, M.L. Kaiser, B. Cecconi, A. Lecacheux, W.M. Farrell, P. Galopeau, J.-C. Gérard, D. Grodent, R. Prangé, M.K. Dougherty, F.J. Crary, An Earthlike correspondence between Saturn's auroral features and radio emission. Nature 433, 722-725 (2005)

S.R. McCandliss, J.B. McPhate, P.D. Feldman, Narcissistic ghosts in Rowland-mounted, concave grating with $\beta=0^{\circ}$ : a cautionary note. Appl. Opt. 37, 5070-5074 (1998)

R.R. Meier, Ultraviolet spectroscopy and remote sensing of the upper atmosphere. Space Sci. Rev. 58, 1-185 (1991)

H. Melin, D.E. Shemansky, X. Liu, The distribution of atomic hydrogen and oxygen in the magnetosphere of Saturn. Planet. Space Sci. 57, 1743-1753 (2009)

D.G. Mitchell, P.C. Brandt, E.C. Roelof, J. Dandouras, S.M. Krimigis, B.H. Mauk, C.P. Paranicas, N. Krupp, D.C. Hamilton, W.S. Kurth, P. Zarka, M.K. Dougherty, E.J. Bunce, D.E. Shemansky, Energetic ion acceleration in Saturn's magnetotail: substormsar Saturn? Geophys. Res. Lett. 32, L20S01 (2005) 
G. Murakami, I. Yoshikawa, Y. Obana, K. Yoshioka, G. Ogawa, A. Yamazaki, M. Kagitani, M. Taguchi, M. Kikuchi, S. Kameda, M. Nakamura, First sequential images of the plasmasphere from the meridian perspective observed by KAGUYA. Earth Planets Space 62, e9-e12 (2010)

G. Murakami, I. Yoshikawa, K. Yoshioka, A. Yamazaki, M. Kagitani, M. Taguchi, M. Kikuchi, S. Kameda, M. Nakamura, Plasmaspheric filament: an isolated magnetic flux tube filled with dense plasmas. Geophys. Res. Lett. 40, 250-254 (2013)

M. Nakamura, K. Yamashita, I. Yoshikawa, K. Shiomi, A. Yamazaki, S. Sasaki, Y. Takizawa, M. Hirahara, W. Miyake, Y. Saito, S. Chakrabarti, Helium observation in the Martian ionosphere by an X-ray ultraviolet scanner on Mars orbiter NOZOMI. Earth Planets Space 51, 61-70 (1999)

C. Paranicas, D.G. Mitchell, E.C. Roelof, B.H. Mauk, S.M. Krimigis, P.C. Brandt, M. Kusterer, F.S. Turner, J. Vandergriff, N. Krupp, Energetic electrons injected into Saturn's neutral gas cloud. Geophys. Res. Lett. 115, L02109 (2007)

C. Paranicas, D.G. Mitchell, S.M. Krimigis, D.C. Hamilton, E. Roussos, N. Krupp, G.H. Jones, R.E. Johnson, J.F. Cooper, T.P. Armstrong, Sources and losses of energetic protons in Saturn's magnetosphere. Icarus 197, 519-525 (2008)

C.C. Porco, P. Helfenstein, P.C. Thomas, A.P. Ingersoll, J. Wisdom, R. West, G. Neukum, T. Denk, R. Wagner, T. Roatsch, S. Kieffer, E. Turtle, A. McEwen, T.V. Johnson, J. Rathbun, J. Veverka, D. Wilson, J. Perry, J. Spitale, A. Brahic, J.A. Burns, A.D. DelGenio, L. Dones, C.D. Murray, S. Squyres, Cassini observes the active South Pole of Enceladus. Science 311, 1393-1401 (2006)

A. Potter, T. Morgan, Discovery of sodium in the atmosphere of Mercury. Science 229, 651-653 (1985)

D.E. Shemansky, P. Matheson, D.T. Hall, H.-Y. Hu, T.M. Tripp, Detection of the hydroxyl radical in the Saturn magnetosphere. Nature 363, 329-331 (1993)

J.R. Spencer, J.C. Pearl, M. Segura, F.M. Flasar, A. Mamoutkine, P. Romani, B.J. Buratti, A.R. Hendrix, L.J. Spilker, R.M.C. Lopes, Cassini encounters Enceladus: background, and discovery of a South Polar hot spot. Science 311, 1401-1405 (2006)

A.J. Steffl, A.I.F. Stewart, F. Bagenal, Cassini UVIS observation of the Io plasma torus. I. Initial results. Icarus 172, 78-90 (2004a)

A.J. Steffl, F. Bagenal, A.I.F. Stewart, Cassini UVIS observation of the Io plasma torus. II. Radial variation. Icarus 172, 91-103 (2004b)

S.A. Stern, D.C. Slater, G.R. Gladstone, E. Wilkeson, W.C. Cash, J.C. Green, D.M. Hunten, T.C. Owen, L. Paxton, NOTE the 825-1110 A EUV spectrum of Venus. Icarus 122, 200-204 (1996)

H. Tadokoro, H. Misawa, F. Tsuchiya, Y. Katoh, A. Morioka, M. Yoneda, Effect of photo-dissociation on the spreading of OH and O clouds in Saturn's inner magnetosphere. J. Geophys. Res. 117, A09226 (2012)

N. Thomas, F. Bagenal, T.W. Hill, J.K. Wilson, in The Io Neutral Clouds and Plasma Torus in Jupiter: Planet, Satellites and Magnetosphere, ed. by Bagenal, Dowling, McKinnon (Cambridge University Press, Cambridge, 2003), pp. 561-591

F. Tsuchiya, M. Kagitani, N. Terada, Y. Kasaba, I. Yoshikawa, G. Murakami, K. Sakai, T. Homma, K. Yoshioka, A. Yamazaki, K. Uemizu, T. Kimura, M. Ueno, Plan for observing magnetospheres of outer planets by using the EUV spectrograph onboard the SPRINT-A/EXCEED mission. Adv. Geosci. 25, 57-72 (2011)

R.J. Vervack Jr., W.E. McClintock, R.M. Killen, A.L. Sprague, B.J. Anderson, M.H. Burger, E.T. Bradley, N. Mouawad, S.C. Solomon, N.R. Izenberg, Mercury's complex exosphere: results from MESSENGER's third flyby. Science 329, 672-675 (2010)

T. Yamazaki, Y. Takizawa, K. Kunieda, K. Yamashita, K. Ikeda, K. Misaki, M. Nakamura, I. Yoshikawa, A. Yamaguchi, Normal incidence multilayer telescope for galactic EUV observation. J. Electron Spectrosc. Relat. Phenom. 80, 299-302 (1996)

A. Yamazaki, S. Tashiro, Y. Nakasaka, I. Yoshikawa, W. Miyake, M. Nakamura, Sounding-rocket observation of O II 83.4-nm emission over the polar ionosphere. Geophys. Res. Lett. 29, 1-4 (2002)

A. Yamazaki, I. Yoshikawa, Y. Takizawa, W. Miyake, M. Nakamura, Feasibility study of the O II 83.4-nm imaging of the ionosphere and magnetosphere. Adv. Space Res. 32, 441-446 (2003)

A. Yamazaki, F. Tsuchiya, T. Sakanoi, K. Uemizu, K. Yoshioka, G. Murakami, M. Kagitani, Y. Kasaba, I. Yoshikawa, N. Terada, T. Kimura, S. Sakai, K. Nakaya, S. Fukuda, S. Sawai, Field-Of-View guiding camera on the HISAKI (SPRINT-A) satellite. Space Sci. Rev. (2014)

I. Yoshikawa, M. Nakamura, M. Hirahara, Y. Takizawa, K. Yamashita, H. Kunieda, T. Yamazaki, K. Misaki, A. Yamaguchi, Observation of He II emission from the plasmasphere by a newly developed EUV telescope on board sounding rocket S-520-19. J. Geophys. Res. 102, 19897-19902 (1997)

I. Yoshikawa, A. Yamazaki, K. Shiomi, M. Nakamura, K. Yamashita, Y. Saito, M. Hirahara, Y. Takizawa, W. Miyake, S. Matsuura, Development of a compact EUV photometer for imaging the planetary magnetosphere. J. Geophys. Res. 106, 26057-26074 (2001)

I. Yoshikawa, T. Murachi, H. Takenaka, S. Ichimaru, Multilayer coating for $30.4 \mathrm{~nm}$. Rev. Sci. Instrum. 76, 066109 (2005) 
I. Yoshikawa, A. Yamazaki, G. Murakami, K. Yoshioka, S. Kameda, F. Ezawa, T. Toyota, W. Miyake, M. Taguchi, M. Kikuchi, M. Nakamura, Telescope of extreme ultraviolet (TEX) onboard SELENE: science from the Moon. Earth Planets Space 60, 407-416 (2008)

I. Yoshikawa, O. Korablev, S. Kameda, D. Rees, H. Nozawa, S. Okano, V. Gnedykh, V. Kottsov, K. Yoshioka, G. Murakami, F. Ezawa, G. Cremonese, The Mercury sodium atmospheric spectral imager for the MMO spacecraft of Bepi-Colombo. Planet. Space Sci. 58, 224-237 (2010a)

I. Yoshikawa, G. Murakami, G. Ogawa, K. Yoshioka, Y. Obana, M. Taguchi, A. Yamazaki, S. Kameda, M. Nakamura, M. Kikuchi, M. Kagitani, S. Okano, W. Miyake, Plasmaspheric EUV images seen from lunar orbit: initial results of the extreme ultraviolet telescope on board the Kaguya spacecraft. J. Geophys. Res. 115, A04217 (2010b)

I. Yoshikawa, T. Homma, K. Sakai, G. Murakami, K. Yoshioka, A. Yamazaki, T. Sakanoi, A. Saito, Imaging observation of the Earth's plasmasphere and ionosphere by EUVI of ISS-IMAP on the international space station. IEEJ Trans. Fundam. Mat. 131, 1006-1010 (2011a)

I. Yoshikawa, K. Yoshioka, G. Murakami, G. Ogawa, M. Ueno, A. Yamazaki, K. Uemizu, S. Kameda, F. Tsuchiya, M. Kagitani, N. Terada, Y. Kasaba, The Exceed mission. Adv. Geosci. 25, 29-41 (2011b)

K. Yoshioka, I. Yoshikawa, F. Tsuchiya, M. Kagitani, G. Murakami, Hot electron component in the Io plasma torus confirmed through EUV spectral analysis. J. Geophys. Res. 116, A09204 (2011)

K. Yoshioka, G. Murakami, I. Yoshikawa, J.-L. Maria, J.-F. Mariscal, N. Rouanet, P.-O. Mine, E. Quemerais, Optical performance of PHEBUS/EUV detector onboard BepiColombo. Adv. Space Res. 49, 1265-1270 (2012)

K. Yoshioka, G. Murakami, A. Yamazaki, F. Tsuchiya, M. Kagitani, T. Sakanoi, T. Kimura, K. Uemizu, K. Uji, I. Yoshikawa, The extreme ultraviolet spectroscope for planetary science, EXCEED. Planet. Space Sci. 85, 250-260 (2013) 